\title{
Severe Complications Related to Caesarean Delivery in Patients with Endometriosis
}

\author{
A. Louise D. Zierau ${ }^{1}$, Jens Langhoff-Roos ${ }^{2}$, Tórur Dalsgaard ${ }^{3}$ and Janne Foss Berlac ${ }^{4}$ \\ ${ }^{1}$ Lungemedicinsk Forskningsenhed, Bispebjerg Hospital, Bispebjerg Bakke, Copenhagen Denmark \\ ${ }^{2,3,4}$ Department of Gynaecology, Juliane Marie Centeret Rigshospitalet, Copenhagen University \\ Hospital Blegdamsvej, Copenhagen, Denmark
}

Correspondence should be addressed to: Dorthe Hartwell; dorthe.hartwell@regionh.dk

Received date: 27 March 2014; Accepted date: 12 May 2015; Published date: 11 February 2016

Academic Editor: Yutaka Osuga

Copyright (C 2016. A. Louise D. Zierau, Jens Langhoff-Roos, Tórur Dalsgaard and Janne Foss Berlac. Distributed under Creative Commons CC-BY 4.0

\begin{abstract}
Introduction: Endometriosis is a chronic inflammatory disease with endometrial-like tissue outside the uterine cavity, which leads to adhesions in the surrounding tissue. In pregnancy, endometriosis-derived ectopic decidua may be induced, and at delivery, the tissues are stretched and complications may occur. We report two cases where caesarean section in women with endometriosis led to severe complications. Case presentations: A 33-year old nullipara had an emergency caesarean section at 37+2 weeks of gestation. Extraction of the infant was complicated due to endometriotic and fibrotic tissue. The postoperative course was complicated by an infected haematoma.A 30-year old nullipara had an emergency caesarean section at $38+3$ weeks of gestation. Excessive bleeding and a large retroperitoneal haematoma complicated the surgery.Conclusion: These two cases emphasize that caesarean delivery in patients with severe endometriosis may be associated with an elevated risk of complications such as intestinal perforation and excessive bleeding due to endometriotic lesions.
\end{abstract}

Key words: Endometriosis, caesarean delivery, haematoma, postoperative complications

\section{Introduction}

Endometriosis is a chronic inflammatory disease characterized by the presence of endometrial-like tissue outside the uterine cavity. The advanced stages of endometriosis result in severe fibrosis and adhesions in the pelvic cavity. The prevalence of endometriosis in women of reproductive age is around 6-10\%, but among women suffering from infertility, the frequency is $35 \%-50 \%$.

With the increasing use of assisted reproduction technology, more women with endometriosis become pregnant.

Several case reports have described severe complications during pregnancy such as spontaneous haemoperitoneum,

Cite this Article as: A. Louise D. Zierau, Jens Langhoff-Roos, Tórur Dalsgaard and Janne Foss Berlac (2016), "Severe Complications Related to Caesarean Delivery in Patients with Endometriosis," International Journal of Case Reports in Medicine, Vol. 2016 (2016), Article ID 620369, DOI: $10.5171 / 2016.620369$ 
spontaneous rupture of subserous uterine veins, and intestinal perforation in women with endometriosis. Epidemiological studies have suggested that endometriosis is associated with preterm birth, antepartal haemorrhage, and an increase in cesarean delivery.

Except for implantation of endometriosis in the surgical scars, there are to our knowledge no reports of complications related to caesarean delivery in patients with endometriosis.

We present two cases of severe complications to caesarean delivery in patients with endometriosis and discuss whether changes in clinical practice may avoid such complications.

\section{Case I}

A 33-year old nullipara pregnant after IVF had an emergency caesarean section due to the slow progress of labour at $37+2$ weeks of gestation.

One year earlier she had a laparotomy at a local hospital. Bilateral endometriomas, endometriotic lesions, and adhesions on the posterior wall of the uterus involving the recto-sigmoid wall were found. The endometriomas were excised but no extensive surgery was performed. A 13+4 weeks an ultrasound scan showed a mass (27 x $48 \times 29 \mathrm{~mm})$ in the bladder wall. Cystoscopy showed typical deep infiltrating endometriosis in the bladder wall but no biopsy was performed. At 19+3 weeks of gestation, she was admitted to the hospital with lower abdominal pain and fever. Magnetic resonance imaging (MRI) showed a slightly dilated small intestine and appendicitis was suspected. At laparotomy, there were signs of diffuse peritonitis and the appendix was removed. Microscopy of the appendix showed acute subserous inflammation and oedema but no signs of primary appendicitis or endometriosis. Her condition improved and she was discharged after seven days. The focus of infection was never identified.

The caesarean delivery was complicated by dense adhesions and difficult access to the uterus. Extraction of the infant was complicated due to the low anterior location of the placenta as well the endometriotic and fibrotic tissue. It was necessary to induce general anesthesia and after 5 minutes the infant was delivered with low Apgar score and admitted to the neonatal intensive care unit. Haemostasis was difficult and the total bleeding volume was $1400 \mathrm{ml}$. The postoperative course was complicated by an infected haematoma sized $4 \times 6 \times 3 \mathrm{~cm}$, above the bladder. She had elevated leucocytes (26 x109/l) and CRP (273 mg/l). The infection was treated conservatively with antibiotics, and the patient was discharged seven days after the caesarean section. The follow-up showed full remission of the haematoma and a persisting asymptomatic endometriotic nodule of $0,6 \times 1,0 \times 2,0 \mathrm{~cm}$ in the bladder wall. She was treated with oral contraceptives.

\section{Case II}

A 30-year old nulliparous pregnant after intrauterine insemination had an emergency caesarean section at $38+3$ weeks of gestation due to the slow progress of labour.

She had a history of endometriosis verified by biopsy from the posterior fornix. At the caesarean section, the posterior wall of the uterus was attached to the left ovary, intestine, and peritoneum.

Mobilization of the uterus resulted in diffuse bleeding from endometriotic lesions on the uterus and the left ovary. Haemostasis was achieved by several sutures and application of TachoSilß. The total bleeding volume was $1500 \mathrm{ml}$ and postoperative hemoglobin was $4.3 \mathrm{mmol} / \mathrm{l}$. The first postoperative day she complained of abdominal pain and a CT-scan showed a retroperitoneal haematoma stretching from the lower part of the left kidney to the bladder, and dilation of both ureters with bilateral hydronephrosis. She developed oedema in the lower extremities and vulva. The hemoglobin fell to $3.6 \mathrm{mmol} / \mathrm{l}$ and an ultrasound scan showed that the haematoma displaced the uterus to the right side and compressed the iliac vessels. Antithrombotic treatment was initiated. On the sixth postoperative day, the patient had increasing pain and fever $\left(38.1^{\circ} \mathrm{C}\right)$. She was treated with antibiotics (Cephalosporin), and received three units of red blood cells. 
On the sixteenth postoperative day she was discharged, and one month later the haematoma had decreased from $20 \times 8 \times 11$ $\mathrm{cm}$ to $11 \times 6 \times 5 \mathrm{~cm}$. At follow up six months later, the symptoms from the haematoma had disappeared.

\section{Discussion}

In general, pregnancy has an alleviating effect on endometriosis related symptoms. However, endometriosis-derived ectopic decidua and dense adhesions due to the endometriosis may cause severe obstetrical complications during pregnancy and in caesarean section as described in the present two cases.

In case I, the delivery of the infant and haemostasis was difficult and resulted in a postoperative infected haematoma. In case II, a large retroperitoneal haematoma aggravated the postoperative course.

Endometriosis is known to be associated with fibrous adhesions as a response to chronic inflammation. During pregnancy, decidualization of the endometriotic lesions changes the structure of the tissue and it becomes highly vascularized and more sensitive. Mobilization of the uterus during caesarean section may therefore be associated with an increased risk of complications including perforation of the intestine and bleeding. Veins imbedded in the adhesions covering the endometriotic lesions may be more likely to rupture - a complication similar to the spontaneous rupture of veins reported during pregnancy and at vaginal delivery Error! Bookmark not defined. in women with endometriosis.

Spontaneous intestinal perforation from decidualized endometriosis in pregnancy has been described. It is suggested that perforation is facilitated by the traction of the enlarged uterus on the strictly adherent sigmoid colon. It seems that mobilization of the uterus during caesarean section in women with severe endometriosis should be managed carefully and preferably; the uterus should remain intra-abdominally during surgery. Until now, only 21 cases of intestinal perforation due to endometriosis have been reported, with our case being the first report of perforation related to caesarean section.

Our cases show that patients suffering from endometriosis and their infants may be at risk of serious complications during caesarean section. To the best of our knowledge, these two cases are the first to be reported. However, with the increasing rate of caesarean sections in patients with endometriosis, these types of complications are likely to occur more frequently in the future. Therefore, it is necessary to raise awareness of the problem and create guidelines on how to manage caesarean delivery in patients with severe endometriosis. We recommend that the obstetrician leaves the uterus intraabdominally during repair, thereby minimizing the stretch of the adhesions to reduce the risk of tissue rupture and bleeding. The risk of recurrence of complications during a second pregnancy in women with endometriosis is still unknown. However, detailed information and guidance is recommended to ensure a quick response to any symptoms.

In Denmark, the gynaecological treatment of severe endometriosis has been centralized to optimize the treatment. We presume that also the obstetric patients with known severe endometriosis would benefit from centralized care.

In conclusion, caesarean delivery in patients with severe endometriosis may be associated with excessive bleeding due to adhesions, dense fibrosis and decidualization of the endometriotic tissue. The rate of complications should be investigated in large population-based studies.

\section{Authorization}

The use of patient data in this project has been approved by The Danish Data Protection Agency [J.no. 2013-41-2150]. The patients have both signed an informed consent.

\section{Financial disclosures}

No financial disclosures have been received by the authors of this case report. 


\section{Conflict of Interest}

There is no conflict of interest regarding this case report.

\section{References}

1. Revised American Society for Reproductive Medicine classification of endometriosis: 1996. Fertil Steril. 1997 May;67(5):817-21.

2. Giudice LC, Kao LC. Endometriosis. Lancet. 2004 Nov 13-19;364(9447):178999

3. Stephansson O, Kieler H, Granath F, Falconer H. Endometriosis, assisted reproduction technology, and risk of adverse pregnancy outcome. Hum Reprod 2009;24:2341-7

4. Granese R. Acute abdomen in pregnancy with endometriosis. Acta Obstet Gynecol Scand 2010;89:844-5

5. Wada S, Yoshiyuki F, Fujino T, Sato C. Uterine vein rupture at delivery as a delayed consequence of laparoscopic surgery for endometriosis: a case report. J Minim Invasive Gynecol 2009;16:510-2

6. Pisanu A, Deplano D, Angioni S, Ambu R, Uccheddu A. Rectal perforation from endometriosis in pregnancy: case report and literature review. World J Gastroenterol 2010;16:648-51

7. Brosens I, Brosens JJ, Fusi L, Al-Sabbagh M, Kuroda K, Benagiano G. Risks of adverse pregnancy outcome in endometriosis. Leuven Institute of Fertility and Embryology, Leuven, Belgium. ivo.brosens@med.kuleuven.be Fertil Steril.
2012 Jul;98(1):30-5. doi: 10.1016/j.fertnstert.2012.02.024. Epub 2012 Mar 3.

8. Nishikawa A, Kondoh E, Hamanishi J, Yamaguchi K, Ueda A, Sato Y, Konishi I. Ileal perforation and massive intestinal haemorrhage from endometriosis in pregnancy: case report and literature review. ) Department of Gynaecology and Obstetrics, Kyoto University, Kyoto, Japan Eur J Obstet Gynecol Reprod Biol. 2013 Sep;170(1):20-4. doi: 10.1016/j.ejogrb.2013.04.018. Epub 2013 Jun 10.

9. Andolf E, Thorsell M, Källén K. Caesarean section and risk for endometriosis: a prospective cohort study of Swedish registries. Division of Obstetrics and Gynaecology, Department of Clinical Sciences, Karolinska Institutet, Danderyd Hospital, Stockholm, Sweden. ellika.andolf@ds.se BJOG. 2013 Aug;120(9):1061-5.

10. Vercellini P, Parazzini F, Pietropaolo G, Cipriani S, Frattaruolo MP, Fedele L. Pregnancy outcome in women with peritoneal, ovarian and rectovaginal endometriosis: a retrospective cohort study. Clinica Ostetrica e Ginecologica, Istituto Luigi Mangiagalli, Università Statale di Milano, and Fondazione IRCCS Ca Granda - Ospedale Maggiore Policlinico, Milan, Italy BJOG. 2012 Nov;119(12):153843.

11. D'Hooghe T, Forman A. European accreditation of endometriosis centers of expertise. Gynecol Obstet Invest. 2013;76(1):1-3. doi: 10.1159/000354330. Epub 2013 Aug 7. 